\title{
Las Perspectivas y Retos de la Antropología Aplicada en el Siglo XXI
}

\section{Marisol Pérez L.}

Licenciatura, Maestría y Doctorado en Antropología Social - Universidad I beroamericana, México D.F.

Profesora-investigadora del Programa de Posgrado en Antropología Social Universidad I beroamericana, México D.F.

marisol.perez@uia.mx

Resumen

El presente artículo reflexiona acerca del rol de la Antropología aplicada en el siglo XXI. Analiza las posibilidades de la antropología aplicada en el contexto actual, sus fortalezas y debilidades frente a los problemas sociales contemporáneos.

\section{Abstract}

This article analizes the role of Applied Anthropology in the twenty-first century. It analyzes the potential of anthropology applied in the present, its strengths and weaknesses faced to contemporary social problems.

Palabras Clave: Antropología Social, Antropología Aplicada, Problemas Sociales, América Latina, Complejidad.

\section{I ntroducción}

Existen múltiples definiciones de antropología aplicada, yo parto para definirla de mi formación en la antropología, con influencias marxistas, de la década de 1960, en México. Con base en ello, defino la antropología aplicada como la práctica profesional que emplea los conocimientos generados por la investigación antropológica, de orden académico, para promover el cambio social y cultural. De acuerdo con Ángel Palerm, mi maestro, este quehacer implica el mostrar la diversidad cultural, el compromiso con los grupos menos favorecidos de la sociedad y la promoción del cambio social y cultural.

Según Palerm y Marieta Baba, mi otra gran maestra de antropología aplicada, norteamericana y decana del departamento de Ciencias Sociales de la Universidad de Detroit, la antropología aplicada debería de ser capaz, no solamente de solucionar problemas, sino también de generar, a través de la investigación aplicada, nuevos conocimientos que permitan explicar procesos sociales. O sea, que no solamente es una disciplina de orden práctico, sino también académico. Otra tarea importante es el estudio sistemático del quehacer de los antropólogos aplicados, con la finalidad de lograr, a partir del análisis de sus experiencias, poder llegar a establecer metodologías y técnicas de trabajo que faciliten el trabajo de los antropólogos aplicados. 
Esta concepción de la antropología aplicada implica que las herramientas básicas de un antropólogo aplicado son la teoría generada a través de la investigación académica, metodologías de investigación muy rigurosas, así como el manejo de herramientas para diseñar soluciones. Esta última condición implica la habilidad, y por qué no, la humildad para trabajar en equipos interdisciplinares que involucren activamente a los grupos afectados por sus proyectos.

En un libro compilado por Baba en 1997 sobre las prácticas de la antropología en distintos países del mundo aparece claramente lo dicho por Barnes acerca de que el quehacer científico es uno social y que por lo tanto está condicionado por la sociedad en que se realiza. Así encontramos que la antropología realizada en los países del centro, como Estados Unidos o Francia, no es la misma que la desarrollada en los países periféricos, en su mayor parte antiguas colonias de los países del centro, como pueden ser México o Nigeria. Esta perspectiva implica entre otras cosas, la discusión sobre las diferencias entre la antropología académica, la que adquiere un carácter distinto en los distintos contextos.

A manera de ejemplo, aquí me referiré, en forma sucinta, a las tendencias de la antropología aplicada en México y los Estados Unidos de América, tradiciones que conozco mejor. En los Estados Unidos se discute ampliamente sobre las relaciones entre ambas antropologías. Por el lado de la academia, se cuestiona el status científico de los trabajos de los antropólogos involucrados en el trabajo profesional aplicado, mientras que aquellos con experiencia profesional, aun en la academia, muestran la estrecha interrelación entre ambos tipos de quehacer. Baba, quien coincide con Palerm menciona que hay varios tipos de antropología aplicada y plantea que el ideal del quehacer antropológico es aquel que además de generar nuevos conocimientos está comprometido con las poblaciones menos favorecidas y el cambio social.

En México esta discusión aparece como banal en cuanto que la disciplina nació como una herramienta ligada con el planteamiento de políticas públicas, especialmente las relacionadas con los grupos desplazados por el desarrollo de tipo capitalista. Según Salomón Nahmad, el gran antropólogo aplicado mexicano, en el libro de Baba, Manuel Gamio, el padre reconocido de la antropología mexicana, pretendía que el conocimiento derivado de la investigación en antropología, debería de producir recomendaciones que promovieran "un desarrollo normal de los pueblos indígenas" y consideraba a la antropología como una forma de "conocimiento político". Dicho en otras palabras, para Gamio, la investigación académica en antropología, debería de servir de base para el diseño de políticas públicas, especialmente las ligadas con los pueblos indígenas. Según Nahmad el estado mexicano empleó sus propuestas teóricas y su proyecto de antropología aplicada para consolidar el nacionalismo y el sistema económico capitalista.

A partir de entonces el desarrollo de la disciplina y el quehacer de los antropólogos en México han estado profundamente vinculados con el estado. Sin embargo, Nahmad en su artículo sobre la antropología aplicada en México, muestra que esta relación no ha sido tan provechosa como se esperaría y en su análisis muestra que, empezando con Gamio, el ideólogo reconocido de la revolución mexicana, las relaciones sociales de los antropólogos con los políticos y funcionarios de estado no han sido fáciles. Esta situación nos remite a la necesidad de estudiar el quehacer y las relaciones sociales de 
los antropólogos, a fin de entender cómo éstas inciden en la difusión de sus conocimientos y en la posibilidad de que realmente la disciplina incida en el desarrollo.

En cuanto a los modelos teóricos empleados, Nahmad menciona que a lo largo del siglo veinte fueron tres los principales, complementarios entre si, en los que se inspiró la antropología aplicada en México para proponer modelos de desarrollo: las teorías de la aculturación, las teorías sobre las clases sociales y la de dependencia y finalmente, la teoría de la evolución multilineal. Sin embargo, menciona que las crisis económica y política por la que atraviesa México representan un reto para los antropólogos, quienes tienen que buscar una nueva teoría que genere proyectos alternativos.

Por otra parte, Claudio Lomnitz en 1996 llamó la atención a los antropólogos mexicanos sobre el hecho de que los cambios en el escenario político y económico mundial han implicado, entre otras cosas, la aparición de nuevos y distintos interlocutores. Dicho en otras palabras ya no es el estado el único posible interlocutor de su trabajo, lo que implica que el modelo propuesto por Gamio de la antropología como un "conocimiento político" tiene que ser replanteado, así como también el modelo de relaciones sociales y profesionales de los antropólogos.

Los antropólogos mexicanos, en grupo, hemos respondido a este reto generando un proyecto de investigación en proceso, dirigido por Esteban Krotz, alumno de Palerm, que lleva el nombre de "Antropología de la antropología" (ADELA), con el objetivo de analizar y cuestionar los procesos y perspectivas de la antropología mexicana.

Como mencionó Lomnitz los cambios ocurridos en el contexto mundial han cambiado profundamente la estructura política y las relaciones sociales no solamente en México, sino en todo el mundo y la investigación antropológica ha dado cuenta ampliamente de ello. Hasta 1930 los modelos de análisis se fincaban en las comunidades, a partir de Redfield en 1930, se empezaron a considerar las interrelaciones entre las comunidades y su contexto. Steward en 1950 resaltó la importancia de los estudios regionales y a partir de 1980 los trabajos de Wallerstein, Wolf y Palerm y otros muchos autores, han dado cuenta de los procesos de mundialización y de lo que ello implica para los distintos pueblos de la tierra.

Estos procesos de mundialización o globalización, como se quieran llamar, plantean retos a todos los antropólogos del mundo, quienes a pesar de sus tradiciones locales, tienen que investigar y explicar fenómenos comunes, como el debilitamiento de los estados, el surgimiento de las nacionalidades e identidades locales, la migración, las comunidades internacionales, las grandes corporaciones y tantos otros fenómenos que caracterizan a la sociedad posmoderna en la que nos ha tocado vivir, descritos entre otros antropólogos por Néstor Garcia Canclini.

Estos fenómenos, muy ligados con el desarrollo del capitalismo financiero y el desarrollo de las tecnologías de la comunicación implican muchas paradojas como un incremento de la centralización del poder en grandes organizaciones internacionales como el Banco Mundial o el Banco Interamericano de Desarrollo, al mismo tiempo que el surgimiento de las famosas organizaciones no gubernamentales (ONG) y la aparición de grupos sociales sin referente geográfico específico como las asociaciones y clubes de migrantes internacionales. Esta paradoja de la centralidad versus la diseminación 
del poder implica una gran fluidez en las relaciones sociales, es decir, mientras que anteriormente los grupos sociales eran estables, hoy día las relaciones cambian continuamente. Al mismo tiempo, encontramos en muchas sociedades, y específicamente en Latino América, un reforzamiento de las relaciones familiares como una búsqueda de raíces estables, en un mundo en constante cambio. Frente al abandono y rechazo de formas de vida e ideologías tradicionales, encontramos la aparición de grupos y asociaciones religiosas fundamentalistas que controlan y dan seguridad a sus integrantes, como puede ser la I glesia de la Luz del Mundo en México.

Estos cambios y paradojas implican retos tanto para el desarrollo de la antropología académica así como para la aplicada. Para Agar implica caos, para otros, como Hackenberg implican cambios en la perspectiva y profundidad de nuestras predicciones y análisis.

Con base en las anteriores consideraciones, a continuación me referiré a algunos retos que aparecen como fundamentales para el futuro de la antropología aplicada:

\section{Conceptuales}

Necesitamos nuevos conceptos que den cuenta de las nuevas unidades de análisis que están apareciendo: redes locales, internacionales, virtuales y de otros tipos, muy ligadas con conceptos como el de capital social, acuñado por un antropólogo, Bourdieu y adoptado por los economistas. Comunidades, locales y extralocales, como por ejemplo las comunidades de migrantes zapotecos u otavalos, de carácter internacional, pero fincadas en la comunidad de origen. Otro concepto importante es el de familia, que cambia según el contexto y cuyas funciones también se han modificado. Así, encontramos la familia "tradicional" compuesta por el padre, la madre y los hijos, pero también familias de mujeres relacionadas por sangre, o no, que se juntan para la reproducción económica y social, y en las que los hombres aparecen solamente como compañeros ocasionales o simplemente como, perdonen la expresión, sementales. También el concepto de religión o iglesia, como es el caso de La Luz del Mundo, que mencioné anteriormente, que es una asociación de carácter religioso que sirve de base comunitaria para migrantes pobres y marginados, y que se ha extendido de Guadalajara, en México, a numerosos países del mundo incluyendo España e Israel.

La definición y análisis de estos conceptos no corresponde exclusivamente a la antropología aplicada, sino muy en especial a la académica, pero la aplicada, requiere de su explicación para poder diagnosticar y dar soluciones válidas a problemas constantes.

\section{Nuevas problemáticas}

Muy ligado a la aparición de nuevos fenómenos sociales y de conceptos está el de nuevas problemáticas. Tomemos como ejemplo el caso de la antropología médica. En la década de 1940 el gran problema era cómo convencer a las poblaciones campesinas y marginales para que permitieran vacunar a sus hijos. Hoy día, que vivimos las consecuencias de las vacunaciones masivas, en que los niños no mueren tanto, las poblaciones crecen y los seres humanos vivimos mas años, aparecen nuevas enfermedades y problemas sociales. También los adelantos de la medicina han permitido la aparición y proliferación de enfermedades antes no conocidas que implican 
problemáticas novedosas, tales como la aceptación social y la necesidad de grupos de apoyo para los enfermos de alzheimer o sida.

En el orden del desarrollo, tenemos el fenómeno del calentamiento global, ligado con la tecnología de los combustibles ligados al petróleo. Por ejemplo, en México, nos aquejan muy diversos problemas ligados con esta temática: desde el problema de la concepción del financiamiento público basado en los recursos petroleros, pasando por la contaminación de las zonas petroleras, hasta el problema de la deforestación de los bosques ligada al fenómeno de los precios del gas. Y no digamos el problema del transporte público urbano que genera emisiones de gases contaminantes y al mismo tiempo, sino se atiende adecuadamente, problemas de orden social, político, económico y de salud pública que afectan a la sociedad en general.

En este mismo orden tenemos problemas relacionados con la conservación de los bosques y de las especies. Mientras que según el criterio de los países centrales y de ciertos grupos sociales mas educados, la desaparición de las especies y de los bosques es un problema central, para muchas comunidades pobres, es un problema de orden secundario y muy ligado con su sustento. Por ejemplo, la mariposa monarca en México. Para los canadienses y estadounidenses la posible extinción de la mariposa monarca implica la no explotación de los bosques en donde llega a invernar; mientras que para las comunidades campesinas dueñas de los bosques, la explotación del bosque es la base de su sustento.

Al mismo tiempo que tenemos esos problemas novedosos, el mundo se sigue enfrentando a la pobreza y a la marginación. Surgen preguntas sobre qué es en realidad la pobreza en el contexto de las sociedades modernas capitalistas ¿Son pobres aquellos que no consumen lo mismo que las sociedades modernas urbanas? Entonces, es posible que nos enfrentemos a conceptos de orden cultural. O bien, ¿Son pobres aquellos que no consumen todo lo que el sistema capitalista requiere para su funcionamiento? Entonces, nos enfrentamos a un problema de índole económica.

Considero que la tarea de explicar, analizar y solucionar estos fenómenos no es tarea exclusiva de los antropólogos. Sin embargo, académicos y aplicados, tenemos la tarea urgente de diagnosticar, explicar y buscar soluciones a estos problemas, al mismo tiempo que tenemos que generar información que enriquezca el conocimiento que permita hacer predicciones, buscando el respeto a la diversidad. Y en esta tarea es fundamental tener presente que los antropólogos involucrados en proyectos aplicados tienen acceso a información privilegiada que puede enriquecer la investigación académica. Y aquí nos enfrentamos a un reto mas de la antropología aplicada: No solamente generar proyectos, sino también conocimiento académico.

\section{Nuevas metodologías}

Según Hackenberg, un viejo sabio estadounidense quien fue editor de la revista Human Organization por muchos años, el espacio de difusión y discusión académica de la "Sociedad para la Antropología Aplicada", el gran reto de la antropología aplicada es el abandono de la metodología clásica. Sin embargo, en una reunión de la misma sociedad, en Portland, Oregon, en 2006, la gran conclusión fue que la principal herramienta de los antropólogos aplicados sigue siendo la etnografía. Y esto requiere de ciertas explicaciones. 
La etnografía clásica en que el investigador se iba al campo al menos por un año, es muy costosa y como es obvio, no responde a los requerimientos de tiempos característicos de las agencias que contratan los servicios de los antropólogos aplicados. Tenemos que recurrir a etnografías rápidas, muchas veces elaboradas por equipos de investigadores y fundamentadas en documentos escritos. Hoy día, existen muchos modelos de la metodología de la "evaluación rápida" utilizada por muchos antropólogos aplicados estadounidenses. Ahora bien, estos modelos o tecnologías de análisis son propiedad intelectual de quienes los desarrollan y no son de fácil acceso. Sin embargo, mi experiencia, sin haber nunca llegado a desarrollar un modelo específico, es que es posible desarrollar evaluaciones rápidas teniendo como base la metodología de la etnografía tradicional. Que, recordemos se basa en un conocimiento teórico y de las fuentes, previo, no hay buena etnografía sin teoría que la sustente.

A pesar de ser la reina de las metodologías de la Antropología y de ser posible realizarla en equipos y en tiempos mas cortos, la etnografía es cara. Se necesitan equipos y programas digitales, así como varios investigadores organizados en equipos. Para complicar aun mas las cosas, las nuevas problemáticas, relacionadas con la globalización implican la etnografía multilocalizada, como la que realizó Kearny estudiando a los migrantes zapotecos en los Estados Unidos. Esto implica tiempo y dinero, así como también el arte del investigador antropólogo para negociar y administrar los recursos necesarios para la investigación.

Según Hackenberg también tenemos que olvidarnos, tanto en la antropología aplicada como en la académica, de las explicaciones de largo alcance. Los tiempos nos hablan de cambios constantes e intempestivos, nuestras explicaciones tienen que sujetarse a esa condición del entorno. Sin embargo, podemos recurrir a otra metodología tradicional de la antropología académica: La comparación. La riqueza de la producción académica permite la comparación de los resultados de los proyectos con otros semejantes. Y la riqueza de las bases de datos y de información digitales permiten hacer la tarea en forma más rápida.

Un aspecto en el que coincido con Hackenberg y que me parece de fundamental importancia, es que en los proyectos se deben incorporar las poblaciones afectadas. En una investigación comparativa que realicé sobre el éxito de los proyectos de antropología aplicada, encontré que los "exitosos" se planteaban, desde su inicio, en colaboración entre el investigador, los demandantes y los usuarios del proyecto, con la finalidad última de buscar soluciones a problemas sentidos por los usuarios.

Un campo de trabajo muy importante y altamente demandado es el de las evaluaciones. Como mencioné anteriormente existen metodologías ya desarrolladas, pero también una gran cantidad de reportes publicados sobre evaluaciones de proyectos de muy diversos tipos. Este es un espacio de trabajo muy rico y de fácil acceso para los antropólogos aplicados, sin embargo, es importante que los resultados de las evaluaciones se expresen no solamente en términos cualitativos, sino también cuantitativos, ya que el lenguaje hegemónico de los tomadores de decisiones son los números. Considero que la traducción de los resultados cualitativos en cuantitativos, para expresarlos en un lenguaje comprensible para los tomadores de decisiones es un reto al que nos tenemos que enfrentar los antropólogos si queremos que nos sigan contratando. 
El sueño o ideal de cualquier antropólogo aplicado es plasmar sus conocimientos en proyectos específicos para la solución de problemas sociales. La experiencia, plasmada en la literatura muestra que además de que en éstos deben de estar incorporados los miembros de los grupos afectados, los proyectos de aplicación práctica tienen que estar diseñados por equipos interdisciplinares.

\section{Aparición de nuevos actores que demandan el trabajo de los antropólogos aplicados}

En el contexto de la globalización y del modelo neoliberal, como mencioné líneas arriba, el estado se ha encogido, los organismos internacionales se han convertido en ejes centrales de decisión y al mismo tiempo, han aparecido grupos y organizaciones, locales e internacionales, muy fluidas y quizá poco organizadas.

Todos estos actores sociales demandan el trabajo de los antropólogos esperando que lleguemos con soluciones a situaciones consideradas como problemáticas por cada uno de ellos. Esta situación implica varios problemas para los antropólogos.

En primer lugar, ya no es claro quien es el contratante mas definido de sus servicios. Por ejemplo en México, el estado, a través de sus numerosas agencias encargadas de atender a los pueblos indígenas, era el principal empleador y formador de antropólogos aplicados. Hoy día el estado contrata menos antropólogos y éstos tienen además la competencia de los sociólogos y representantes de otras profesiones. Además el estado muchas veces contrata estudios por fuera, es decir con universidades, centros de investigación, despachos privados de consultoría y organizaciones no gubernamentales especializadas. Esto implica que las posibles fuentes de empleo de los antropólogos aplicados de han diversificado, y que los antropólogos han tenido que cambiar su mentalidad y aceptar que su campo de acción no es solamente el estado y las universidades, sino también los despachos de consultoría, las organizaciones no gubernamentales, las empresas, las corporaciones y aun los despachos de mercadotecnia. Por ejemplo en Intel, Motorola y General Motors en los Estados Unidos tienen antropólogos de planta. En México y los Estados Unidos encontramos muchos antropólogos realizando estudios de mercado en agencias publicitarias y despachos de consultoría.

Esta situación de empleo de los antropólogos implica, además de un cambio de mentalidad, una diversificación de sus conocimientos. Un antropólogo hoy día, no solamente tiene que saber sobre grupos indígenas, sino también sobre industria, sociedades urbanas, tecnología, mercados y publicidad, entre otras cosas.

Otra consecuencia de esta situación se traduce en un dilema de identidad y ético, al menos entre los antropólogos mexicanos. La sociedad mexicana y ellos mismos se ven a si mismos como los especialistas en indios y campesinos y muchos estudiantes llegan a la universidad con el afán de apoyar a las comunidades indígenas, desde el estado. Sin embargo, la realidad es distinta y muchas veces tienen que trabajar en instancias y organizaciones privadas, muy ajenas a la problemática indígena. Esto también implica dilemas de orden ético-político: ¿Cómo un antropólogo va a trabajar para instancias y actores considerados tradicionalmente como los explotadores de los indígenas y de los pobres? 
Sin embargo, las mismas comunidades, iglesias, sindicatos y organizaciones no gubernamentales ligados con los grupos menos favorecidos de la sociedad también demandan los servicios profesionales de los antropólogos. Pero, muchas veces estas organizaciones no tienen los recursos suficientes para pagar los servicios profesionales de los antropólogos. Y ahí se presentan otros dilemas de orden ético-práctico ¿Cómo cobrar por los servicios a la gente pobre, si el profesional también requiere de recursos para sobrevivir?

Y esto nos acerca a otra problemática presente en el ejercicio profesional de los antropólogos. ¿Cómo conseguir recursos para desarrollar proyectos de antropología aplicada, fuera del orden gubernamental? Hoy día hay numerosas agencias y organizaciones con fondos para apoyar proyectos de desarrollo de muy distintos tipos. Pero es necesario conocerlos, saber acceder a ellos y comunicarse debidamente. Existen programas y bases de datos digitales con la información básica necesaria, sin embargo estos programas son caros y es necesario aprender a manejarlos. También es necesario aprender a solicitar los fondos, llenar los formatos y plantear los proyectos en los términos empleados por las agencias de financiamiento. Asimismo es necesario plantear los resultados en los términos esperados por las agencias. Esto implica que muchas veces se tienen que escribir varios reportes con distintos fines.

Ante esta situación las escuelas y maestros de antropología tenemos la obligación de plantearnos la pregunta ¿Hemos preparado a nuestros egresados para estos retos? Honestamente creo que no.

\section{La ética}

Un tema presente en la discusión de los antropólogos aplicados estadounidenses es la ética. En México esta discusión se inició en la década de 1970 alrededor de las responsabilidades y obligaciones de los antropólogos extranjeros que realizaban investigación en México. Aparentemente esta discusión también ha estado presente en distintos países de Latino América y en los Estados Unidos, especialmente a raíz del proyecto Camelot. No tengo información acerca de este tema entre los antropólogos de otros países.

En Brasil la sociedad de antropólogos brasileños tiene un código de ética, también la sociedad de antropología americana. El Colegio de Etnólogos y Antropólogos Sociales de Méxcio ha realizado algunas reuniones en torno a la temática, pero aun no hemos logrado concretar nuestros puntos de vista en un código específico.

Hoy día estoy en proceso de editar un número de la revista Cuicuilco, el órgano de difusión académica de la Escuela Nacional de Antropología e Historia de México, sobre las relaciones entre la ética y la Antropología Aplicada, a petición expresa de la revista. Los investigadores participantes de varios países muestran que son muchas las preguntas en torno al tema, pero éstas se centran especialmente en torno a las relaciones entre los antropólogos, las organizaciones que demandan los proyectos y los usuarios o sujetos de los proyectos. También prevalece claramente la idea de que el compromiso de la Antropología es básicamente con la diversidad y con los pueblos menos favorecidos. En su texto Carolyn Fluehr-Lobban, miembro de la comisión de ética de la Asociación de Antropología Americana concluye que a última instancia, 
entre antropólogos, una investigación ética es una buena investigación, que de cuenta de la realidad y de la diversidad.

Mi reflexión es que este es un campo en el que aun tenemos mucho que investigar y reflexionar los antropólogos aplicados latinoamericanos. RM

\section{Bibliografía}

BABA, Marietta. 2000. "Theories of Practice in Anthropology: A Critical Approach" In The Unity of Theory and Practice in Anthropology: Rebuilding a Fractured Synthesis. NAPA BULLETIN, No. 18 pp. 17-44.

BABA, Marietta y Carole HILL. 1997. "Introduction”. En The Global Practice of Anthropology. Williamsburg, Vi.: Studies in Third World Societies. Pp: 1-24.

BARNES, Barry and David EDGE. 1982. Science in Context. Milton Keynes: The Open University Press.

HACKENBERG, Robert A. y Beverly H. Hackenberg. 2004. “Notes Toward a New Future: Applied Anthropology in Century XXI" Human Organization, Vol 63, No. 4.

NAHMAD, Salomón. 1997."Mexican Applied Anthropology: From Founder Manuel Gamio to Contemporary Movements. En Baba M. (ed.) The Global Practice of Anthropology. Williamsburg, Vi.: Studies in Third World Societies. PP: 229-244.

PALERM VICH, Ángel. 1987. Teoría Etnológica. Querétaro: Universidad Autónoma de Querétaro

PÉREZ LIZAUR, Marisol. .- “Antropología aplicada en México: Temas por investigar y metodologías novedosas" Revista Cuicuilco. No. 35, 2005, 59-78 